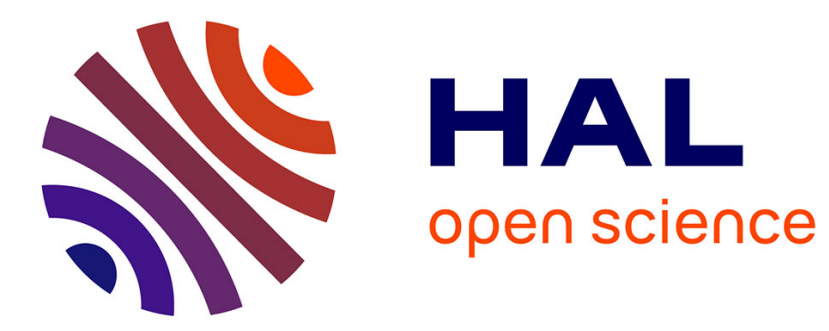

\title{
On the necessity of the solvable conditions of the typical boundary value problems for quasilinear hyperbolic systems
}

\author{
T.T. Li, W.T. Yu
}

\section{To cite this version:}

T.T. Li, W.T. Yu. On the necessity of the solvable conditions of the typical boundary value problems for quasilinear hyperbolic systems. RR-0075, INRIA. 1981. inria-00076486

\section{HAL Id: inria-00076486 \\ https://hal.inria.fr/inria-00076486}

Submitted on 24 May 2006

HAL is a multi-disciplinary open access archive for the deposit and dissemination of scientific research documents, whether they are published or not. The documents may come from teaching and research institutions in France or abroad, or from public or private research centers.
L'archive ouverte pluridisciplinaire HAL, est destinée au dépôt et à la diffusion de documents scientifiques de niveau recherche, publiés ou non, émanant des établissements d'enseignement et de recherche français ou étrangers, des laboratoires publics ou privés. 


\section{Rapports de Recherche}

$$
\mathrm{N}^{\circ} 75
$$

\section{ON THE NEGESSITY OF THE SOLVABLE CONDITIONS OF THE TYPICAL BOUNDARY VALUE PROBLEMS FOR QUASILINEAR HYPERBOLIC SYSTEMS}

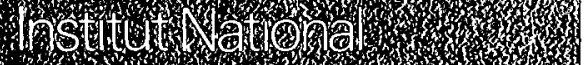

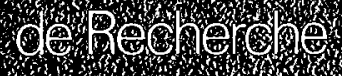
6. E.OA A

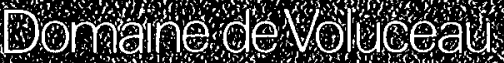

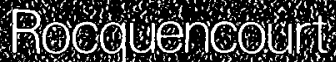
2.1.5. \% 7. (8) 020

\author{
LI Ta-Tsien \\ YU Wen-Tzu
}


ON THE NECESSITY OF THE SOLVABLE CONDITIONS OF THE TYPICAL BOUNDARY VALUE PROBLEMS FOR QUASILINEAR HYPERBOLIC SYSTEMS

$$
\begin{aligned}
& \text { LI Ta - Tsien (LI Daqian) } \\
& \text { and } \\
& \text { YU Wen - Tzu (YU Wenci) }
\end{aligned}
$$

RESUME : Dans cet article, on étudie la nécessité des conditions de solvabilité du problème aux limites typique pour les systèmes hyperboliques quasi-linéaires à deux variables sur un domaine angulaire.

Ces conditions, déjà introduites dans [1] comme conditions suffisantes, signifient que les valeurs au sommet de toutes les dérivées de la solution peuvent être déterminées de manière unique à partir des données.

\section{ABSTRACT :}

In this article we discuss the necessity of the conditions for solvability of the typical boundary value problem for quasi-linear hyperbolic systems in two variables on an angular domain. These conditions, already introduced as sufficient conditions for solvability in [1], mean that all the derivatives of the solution can be determined uniquely at the vertex from the data. 
ON THE NECESSITY OF THE SOLVABLE CONDITIONS OF THE TYPICAL BOUNDARY VALUE PROBLEMS FOR QUASILINEAR HYPHRBOLIC SYSTEFMS

\author{
by \\ Li Daqian* and Yu Wenci** \\ (Li Ta-Tsien) (Yu Wen-Tzu) \\ Fudan University, Shanghai \\ The People's Republic of China
}

\begin{abstract}
Some solvable conditions have been derived to ensure the existence and the uniqueness of the $C^{\infty}$ solution for the typical boundary problem on a local angular region for quasilinear hyperbolic systems in two varia.. bles [1]. These solvables conditions mean that, under the formulation of the typical boundary problem, the all order derivatives of the solution can be determined uniquely at the vertex. The main purpose of this paper is to show that these solvable conditions are also necessary. In other words, if these solvable conditions fail to hold, then the boundary value problem will either have no solution or have infinite number of solutions.

1. Description of the typical boundary value problem. We consider
\end{abstract} the following typical boundary value problem on an angular region $R(\delta)=\{(t, x) \mid 0 \leqq t \leqq \delta, 0 \leqq x \leqq t\}$, in which $u=\left(u_{1}, u_{2}, \ldots u_{n}\right)^{T}$ stands for an unknown vector function :

$$
\begin{cases}G(t, x, u) \frac{\partial u}{\partial t}+\lambda(t, x, u) G(t, x, u) \frac{\partial u}{\partial x}=\mu(t, x, u), \\ u_{r}=G_{r}(t, u) & (r=1,2, \ldots, m) \text { on } x=t, \\ u_{s}=G_{s}(t, u) & (s=m+1, \ldots, n) \text { on } x=0,\end{cases}
$$


where $6: R^{2+n} \rightarrow R^{n \times n}, \mu: R^{2+n} \rightarrow R^{n}, G=\left(G_{1}, G_{2}, \ldots, G_{n}\right)^{T}: R^{n+1} \rightarrow R^{n} \quad$ and $\lambda: \mathrm{R}^{2+\mathrm{n}} \rightarrow \mathrm{R}^{\mathrm{n} \times \mathrm{n}}$ with

$$
\lambda=\operatorname{diag}\left\{\lambda_{1}, \ldots, \lambda_{n}\right\}
$$

Assumption 1. $6, \lambda, \mu, G$ are all $C^{\infty}$ functions . Assumption 2. There is a unique $u(0,0)$ satisfying

$$
u(0,0)=G(0, u(0,0)) \text {. }
$$

Assumption $3 . \quad 6(0,0, u(0,0))=I$, i.e. the identity matrix .

Assumption 4. For $r=1, \ldots, m$ and $s=m+1, \ldots, n$,

$$
\lambda_{r}(0,0, u(0,0))<0<1<\lambda_{s}(0,0, u(0,0)) \text {. }
$$

Assumption 5. Let $\oplus_{0}$ stands for the characterizing matrix [2] of the problem (1) - (3), i.e.

$$
\Theta_{0}=\left(\frac{\partial G}{\partial u}(0, u(0,0))\right. \text {, }
$$

then the both principal submatrices of $\Theta_{0}$ either composed by the first $m$ rows and the first $m$ columns or the last $(n-m)$ rows and the last (n-m) columns are zero.

Usually we may assume that $G_{r}(r=1, \ldots, m)$ does not depend on $u_{s}$ $(s=m+1, \ldots, n)$ and $G_{s}(s=m+1, \ldots, n)$ does not depend on $u_{r}(r=1, \ldots, m)$. Obviously in this case assumption 5 is certainly satisfied.

Setting

$$
\sigma_{0}=\operatorname{diag}\left\{\sigma_{1}, \ldots, \sigma_{n}\right\}
$$

where

$$
\left\{\begin{array}{cc}
\sigma_{r}=\frac{\lambda_{r}(0, u(0,0))}{\lambda_{r}(0, u(0,0))-1} & (r=1, \ldots, m), \\
\sigma_{s}=\frac{\lambda_{s}(0, u(0,0))-1}{\lambda_{s}(0, u(0,0))} & (s=m+1, \ldots, n),
\end{array}\right.
$$

from assumption 4 we know that

$$
0<\theta_{\ell}<1 \quad(\ell=1, \ldots, n) \text {. }
$$

The solvable condition obtained in [2] is that the minimal characterizing number of this boundary value problem is less than one, i.e.

$$
\left|\Theta_{0}\right| \triangleq \inf _{\gamma}\left\|\gamma G \gamma^{-1}\right\|_{\infty}<1,\left(\gamma=\operatorname{diag}\left\{\gamma_{1}, \ldots, \gamma_{n}\right\}, \gamma_{i} \neq 0\right),
$$

where $\|.\|_{\infty}$ stands for infinite power norm of a matrix. On the ground of this result, we have obtained the weakened solvable conditions in [1] as 
follows :

$$
\operatorname{det}\left|I-\oplus_{0} \sigma_{0}^{k}\right| \neq 0 \quad(k=1,2,3, \ldots) \text {, }
$$

where $g_{0}^{k}$ is the power of $\sigma_{0}$ up to $k$. In the following we are going to verify the necessity of conditions (12).

2. The first augmented problem of the problem (1)-(3). Here we sketch the method in [1] with some different notations. Let

(13) $D=\operatorname{diag}\left\{D_{1}, \ldots, D_{n}\right\}=\operatorname{diag}\left\{\frac{\partial}{\partial t}+\frac{\partial}{\partial x}, \ldots, \frac{\partial}{\partial t}+\frac{\partial}{\partial x}, \frac{\partial}{\partial t}, \ldots, \frac{\partial}{\partial t}\right\}$, where the first m diagnal elements are $\frac{\partial}{\partial t}+\frac{\partial}{\partial x}$, and the next diagnal elements are $\frac{\partial}{\partial t}$. Similarly, let

$$
E=\operatorname{diag}\left\{\frac{\partial}{\partial t}, \ldots, \frac{\partial}{\partial t}, \frac{\partial}{\partial t}+\frac{\partial}{\partial x}, \ldots, \frac{\partial}{\partial t}+\frac{\partial}{\partial x}\right\} .
$$

Then we define that

$$
\left\{\begin{array}{l}
u^{1}=D u \\
v^{1}=E u
\end{array}\right.
$$

Now we are going to derive the new system satisfied by $u^{1}$ supposing $u$ to be a $\mathrm{C}^{\infty}$ solution of the problem (1) - (3), Assuming that

$$
\left\{\begin{array}{l}
I_{1}=\operatorname{diag}\{1, \ldots, 1,0, \ldots, 0\}, \\
I_{2}=\operatorname{diag}\{0, \ldots, 0,1, \ldots, 1\},
\end{array}\right.
$$

where the first parts in the above two expressions contain m elements, and the next parts contain $(n-m)$ elements. It is obvious that

$$
I \frac{\partial}{\partial t}=I_{2} D+I_{1} E
$$

and

$$
I \frac{\partial}{\partial x}=\left(I_{1}-I_{2}\right)(D-E) \text {. }
$$

So the original system (1) turns to be

$$
\left[\lambda 6 I_{1}+(I-\lambda) 6 I_{2}\right] D u+\left[(I-\lambda) 6 I_{1}+\lambda \zeta I_{2}\right] \mathrm{E} u=\mu .
$$

Because of assumptions 3 and 4 , the coeffictent matrix of $\mathbf{E u}$ in the above algebraic system is nonsingular in the neighborhood of the origin, therefore $v^{1}=E u$ can be solved out in terms of $u^{1}=D u$ in the corresponding region as follows

$$
v^{1}=a(t, x, u) u^{1}+b(t, x, u)
$$


where

$$
\begin{aligned}
& a=-\left[(I-\lambda) 6 I_{1}+\lambda 6 I_{2}\right]^{-1} \cdot\left[\lambda 6 I_{1}+(I-\lambda) 6 I_{2}\right], \\
& b=\left[(I-\lambda) 6 I_{1}+\lambda 6 I_{2}\right]^{-1} \mu .
\end{aligned}
$$

Also, from (22), (9) and assumption 3, we have that

$$
a(0,0, u(0,0))=\sigma_{0}=\operatorname{diag}\left\{\sigma_{1}, \ldots, \sigma_{n}\right\} \text {. }
$$

By applying every operator $D_{l}$ defined in (13) to the $l-t h$ equation of system (1), substituting (21) into it, and taking them together [1], we get the system of equations satisfied by $u^{1}=D u$ as follows

$$
\sigma^{1}(t, x, u) \frac{\partial u^{1}}{\partial t}+\lambda(t, x, u) \sigma^{1}(t, x, u) \frac{\partial u}{\partial x}=\mu^{1}\left(t, x, u, u^{1}\right),
$$

where

and $\mu^{1}$ is determined by $\zeta, \lambda, \mu$.

$$
6^{1}=6+\left(I_{1} 6 I_{2}+I_{2} 6 I_{1}\right)(a-I)
$$

For the boundary value conditions for $u^{1}$, take the differentiations of (2) and (3) with respect to $t$, and applying (21), we get that

(27) $u_{r}^{1}=G_{r}^{1}\left(t, u, u^{1}\right) \triangleq \frac{\partial G_{r}}{\partial u}(t, u)\left(I_{1}+I_{2} a(t, t, u)\right) u^{1}+\beta_{r}^{1}(t, u)(r=1, \ldots, m)$ on $x=t$, (28) $u_{s}^{1}=G_{s}^{1}\left(t, u, u^{1}\right) \triangleq \frac{\partial G_{s}}{\partial u}(t, u)\left(I_{2}+I_{1} a(t, o, u)\right) u^{1}+\beta_{s}^{1}(t, u)(s=m+1, \ldots, n)$ on $x=0$, where $\frac{\partial G_{r}}{\partial u}$ is taken as a row vector, and $\beta^{1}=\left(\beta_{1}^{1}, \ldots, \beta_{n}^{1}\right)^{T}$ is as follows :

$$
\begin{cases}\beta_{r}^{1}=\frac{\partial G_{r}}{\partial u}(t, u) I_{2} b(t, t, u)+\frac{\partial G_{r}}{\partial t}(t, u) & (r=1, \ldots, m), \\ \beta_{s}^{1}=\frac{\partial G_{B}}{\partial u}(t, u) I_{1} b(t, o, u)+\frac{\partial G_{B}}{\partial t}(t, u) & (s=m+1, \ldots, n) .\end{cases}
$$

Besides, by the definition (15) of $u^{1}$, we get the boundary conditons for $u$ as follows :

$$
\begin{array}{ll}
u_{r}=u_{r}(0,0)+\int_{0}^{t} u_{r}^{1}(\tau, \tau) d \tau & (r=1, \ldots, m) \text { on } x=t, \\
u_{s}=u_{s}(0,0)+\int_{0}^{t} u_{s}^{1}(\tau, 0) d \tau \quad(s=m+1, \ldots, n) \text { on } x=0 .
\end{array}
$$

The new boundary value problem (1), (25), (27), (28), (30) and (31) for $u$ and $u^{1}$ is called the first augmented problem of the original problem (1) $-(3)$. 
3. The general augmented problems - By using the above method, similarly we may get a new system of equations and boundary value conditions satisfied by $u^{0}=u, u^{1}=D u, \ldots, u^{K}=D^{K} u$, and call it the $k$-th augmented problem of the original problem (1)-(3).

The equations of the $\mathrm{K}$-th augmented problem are as follows

$$
\zeta^{k}\left(t, x, u^{o}\right) \frac{\partial u^{k}}{\partial t}+\lambda\left(t, x, u^{o}\right) \zeta^{k}\left(t, x, u^{o}\right) \frac{\partial u^{k}}{\partial x}=\mu^{k}\left(t, x, u^{o}, u^{1}, \ldots, u^{k}\right),
$$

where $k=0,1, \ldots, K$. The coefficient matrices $b^{k}\left(t, x, u^{0}\right)$ in the above system are determined inductively as follows : $\zeta^{\circ}=\zeta, a^{\circ}=a$ and

$$
\left\{\begin{array}{l}
\zeta^{k}=\zeta^{k-1}+\left(I_{1} \zeta^{k-1} I_{2}+I_{2} \zeta^{k-1} I_{1}\right)\left(a^{k-1}-I\right) \\
a^{k-1}=-\left[(I-\lambda) \zeta^{k-1} I_{1}+\lambda \zeta^{k-1} I_{2}\right]^{-1}\left[\lambda \cdot \zeta^{k-1} I_{1}+(I-\lambda) \zeta^{k-1} I_{2}\right]
\end{array}\right.
$$

where $k=1,2, \ldots, k$. These inductive relations are essentially the same as (26) and (22), because, among the equations of (32), the proces of getting $k$ - th equations from $(k-1)$ - th equations for any $k$ is essentially the same as in the case $k=0$. Besides, the details of $\mu^{k}$ are omjtted here.

The boundary value conditions for $u^{K}$ are as follows

$$
\begin{aligned}
& u_{r}^{K}=G_{r}^{K}\left(t, u^{0}, u^{1}, \ldots, u^{K}\right) \\
& \triangleq \frac{\partial G_{r}}{\partial u}\left(t, u^{0}\right)\left[\prod_{i=0}^{K-1}\left(I_{1}+I_{2} a^{i}\left(t, t, u^{0}\right)\right)\right] u^{K}+\beta_{r}^{K}\left(t, u^{0}, \ldots, u^{K-1}\right) \\
& u_{s}^{K}=G_{s}^{K}\left(t, u^{0}, u^{1}, \ldots, u^{K}\right) \quad(r=1, \ldots, m) \text { on } x=t, \\
& \triangleq \frac{\partial G_{s}}{\partial u}\left(t, u^{0}\right)\left[\prod_{i=0}^{K-1}\left(I_{2}+I_{1} a^{i}\left(t, o, u^{0}\right)\right)\right] u^{K}+\beta_{s}^{K}\left(t, u^{\circ}, \ldots, u^{K-1}\right) \\
& \quad(B=m+1, \ldots, n) \text { on } x=0,
\end{aligned}
$$

where $\beta^{k}=\left(\beta_{1}^{k}, \ldots, \beta_{n}^{k}\right)^{T}(k=1, \ldots, K)$ and hence $G^{k}=\left(G_{1}^{k}, \ldots, G_{n}^{k}\right)^{T}$ are determined by induction on $k, \beta^{1}$ is shown in (29), and $\beta^{k+1}$ goes out after taking differentiation of $\mathrm{G}^{\mathrm{k}-1}=\left(\mathrm{G}_{1}^{\mathrm{k}-1}, \ldots, \mathrm{G}_{\mathrm{n}}^{\mathrm{k}-1}\right)^{\mathrm{T}}$ with respect to $t$, and substituting the expression of $E u^{i-1}$ by $u^{i}=D u^{i-1}(i=1, \ldots, k)$ inside. Here we omit the details of this procedure.

The boundary value conditions for $\mathrm{u}^{0}, \ldots, \mathrm{u}^{\mathrm{K}-1}$ are as follows

$$
\begin{array}{ll}
u_{r}^{k}=u_{r}^{k}(0,0)+\int_{0}^{t} u_{r}^{k+1}(\tau, \tau) d \tau & (r=1, \ldots, m) \text { on } x=t, \\
u_{s}^{k}=u_{s}^{k}(0,0)+\int_{0}^{t} u_{s}^{k+1}(\tau, 0) d \tau & (s=m+1, \ldots, n) \text { on } x=0,
\end{array}
$$


where $k=0,1, \ldots, k-1$ and $u^{k}(0,0)$ satisfies the compatibility condition

$$
u^{k}(0,0)=G^{k}\left(0, u^{o}(0,0), \ldots, u^{k}(0,0)\right) \text {, }
$$

where $k=0,1, \ldots, k-1$ and $G^{\circ} \triangleq G$.

by $\left(A_{K}\right)$ we denote the $K-$ th augmented problem consisting of (32), (34) - (37) for any determined $u^{k}(0,0)(k=0,1, \ldots, K-1)$. The following Lemmas will show some interesting properties of $\left(A_{K}\right)$. Jaing $(33)$, by induction we may get the following

Lemma 1. Under assumption 3, i.e. $6^{0}(0,0, u(0,0))=I$, we have that

$$
\begin{aligned}
& \zeta^{k}(0,0, u(0,0))=I, \\
& a^{k-1}(0,0, u(0,0))=\sigma_{0},
\end{aligned}
$$

where $k=1,2, \ldots, k$ and $\sigma_{0}$ is given by (8).

Lemma 2. For the Jacobian matrix $Q_{K}$ of the right sides of the boundary value conditions (34) and (35) in $\left(A_{K}\right)$ at the origin, i.e. (40) $Q_{k}=I_{1} \otimes_{0} \prod_{i=0}^{K-1}\left(I_{1}+I_{2} a^{i}(0,0, u(0,0))\right)+I_{2}{ }_{0} \prod_{i=0}^{K-1}\left(I_{2}+I_{1} a^{i}(0,0, u(0,0))\right)$, where $\otimes_{0}=\left(\frac{\partial G}{\partial u}(0, u(0,0))\right)$, if assumption 5 holds, i.e.

$$
I_{1} \oplus_{0} I_{1}+I_{2} \oplus_{0} I_{2}=0
$$

then it follows that

$$
\Theta_{\mathrm{K}}=\Theta_{0} \sigma_{0}^{\mathrm{K}} \text {, }
$$

where $\sigma_{0}$ is given by (8), and $\sigma_{0}^{K}$ means the power of $\sigma_{0}$ up to $\mathbf{K}$.

Proof - Noticing (39), expanding the right side of (40), and applying (41) and $I_{1} I_{2}=I_{2} I_{1}=0$, we may get that

$$
\oplus_{\mathrm{K}}=I_{1} \oplus_{0}\left(I_{1}+I_{2} \sigma_{0}\right)^{\mathrm{K}}+I_{2} \oplus_{0}\left(I_{2}+I_{I} \sigma_{0}\right)^{K}=\left(I_{1} \oplus_{0} I_{2}+I_{2} \oplus_{0} I_{1}\right) 0_{0}^{K}
$$

again by (41), we obtain

so (42) is proved.

$$
I_{1} \otimes_{0} I_{2}+I_{2} \oplus_{0} I_{1}=\left(I_{1}+I_{2}\right) \oplus_{0}\left(I_{1}+I_{2}\right)=\oplus_{0},
$$

Lemma 3. Suppose that assumptions $1-5$ hold, and $u$ is a $c^{\infty}$ solution of the original problem $\left(A_{0}\right)$, then $\left(u, D u, \ldots, D^{K} u\right)$ is a $c^{\infty}$ solution of the augmented problem $\left(A_{K}\right)$. On the reverse, if $\left(u^{0}, u^{1}, \ldots, u^{K}\right)$ 
is a $C^{\infty}$ solution of the augmented problem $\left(A_{K}\right)$ with the compatibility conditions (38) being satisfied, then $u^{0}$ is a solution of $\left(A_{0}\right)$.

Proof. The first half of this Lemma is obvious consequence from the definition of $\left(A_{K}\right)$. In order to prove the second half, we use induction on $K$. When $K=0$, the conclusion is trivial. Now assume that the conclusion for $\mathrm{K}-1$ is right, and then we prove the conclusion for $K$. Thus we have the solution $\left(\mathrm{u}^{0}, \ldots, \mathrm{u}^{\mathrm{K}-1}, \mathrm{u}^{\mathrm{K}}\right)$ of $\left(\mathrm{A}_{\mathrm{K}}\right)$, and we need to show that $\left(\mathrm{u}^{0}, \ldots, \mathrm{u}^{\mathrm{K}-1}\right)$ is the solution of $\left(\mathrm{A}_{\mathrm{K}-1}\right)$. The only gap here is to verify that $u^{K-1}$ satisfies the boundary value conditions of the form (34) and (35) but for the case $u^{\mathrm{K}-1}$ instead of $u^{\mathrm{K}}$.

Introducing $\overline{\mathrm{u}}^{\mathrm{K}}=\mathrm{D} \mathrm{u}^{\mathrm{K}-1}$, and applying the differential operator $\mathrm{D}$ on the equations of $\mathrm{u}^{\mathrm{K}-1}$ in (32), we know that $\mathrm{u}^{-\mathrm{K}}$ satifies the corresponding equations of $\mathrm{u}^{\mathrm{K}}$ in (32), in which $\mathrm{u}^{\circ}, \ldots, \mathrm{u}^{\mathrm{K}-1}$ can be considered as known functions here. Furthermore, taking differentiation on the conditions of (36) and (37) in the case $k=\mathrm{K}-1$, we get that

$$
\begin{array}{ll}
\mathrm{u}_{r}^{\mathrm{K}}=D u_{r}^{\mathrm{K}-1}=u_{r}^{\mathrm{K}} & (\mathrm{r}=1, \ldots, m) \text { on } \mathrm{x}=t, \\
\bar{u}_{\mathrm{s}}^{\mathrm{K}}=\mathrm{D} \mathrm{u}_{\mathrm{g}}^{\mathrm{K}-1}=\mathrm{u}_{\mathrm{g}}^{\mathrm{K}} \quad(\mathrm{s}=\mathrm{m}+1, \ldots, \mathrm{n}) \text { on } \mathrm{x}=0 .
\end{array}
$$

So, by the uniqueness theorem, $\mathrm{u}^{\mathrm{K}}=\mathrm{D} \mathrm{u}^{\mathrm{K}-1}$ and $\mathrm{u}^{\mathrm{K}}$ are the same on the relevant region, i.e.

$$
\mathrm{u}^{\mathrm{K}}=\mathrm{D} \mathrm{u}^{\mathrm{K}-1}
$$

Now, from (47) and the procedure of derivation of $G^{K}=\left(G_{1}^{K}, \ldots G_{n}^{K}\right)^{T}$ in (34) and (35), we may rewrite (34) and (35) into

$$
\begin{aligned}
& \frac{d}{d t} u_{r}^{K-1}=\frac{d}{d t} G_{r}^{K-1}\left(t, u^{\circ}, \ldots, u^{K-1}\right) \quad(r=1, \ldots, m) \text { on } x=t, \\
& \frac{d}{d t} u_{s}^{K-1}=\frac{d}{d t} G_{s}^{K-1}\left(t, u^{\circ}, \ldots, u^{K-1}\right) \quad(s=m+1, \ldots, n) \text { on } x=0 .
\end{aligned}
$$

Thus, by intergrating (48) and (49) with respect to $t$ and using the compatibility conditions (38) in the case $\mathrm{k}=\mathrm{K}-1$, we obtain

$$
\begin{aligned}
& u_{r}^{\mathrm{K}-1}=G_{r}^{\mathrm{K}-1}\left(t, u^{0}, \ldots, \mathrm{u}^{\mathrm{K}-1}\right) \quad(\mathrm{r}=1, \ldots, \mathrm{m}) \text { on } \mathrm{x}=\mathrm{t}, \\
& u_{s}^{\mathrm{K}-1}=G_{s}^{\mathrm{K}-1}\left(t, u^{\circ}, \ldots, u^{\mathrm{K}-1}\right) \quad(\mathrm{s}=\mathrm{m}+1, \ldots, \mathrm{n}) \text { on } \mathrm{x}=0 .
\end{aligned}
$$

Therefore, Lemma 3 is proved. 


\section{Conclusion}

Theorem 1. Under assumptions 1-5, there exists a unique local $c^{\infty}$ solution $u$ for the typical boundary value problem (1) - (3) iff

$$
\operatorname{det}\left(I-\oplus_{0} \sigma_{0}^{k}\right) \neq 0
$$

holds for every positive integer $k$.

Proof The sufficiency of conditions (52) has already been shown in [1], the basic idea is to use the result in [2] on an augmented problem $\left(A_{K}\right)$ for $K$ large enough. Now we prove the necessity of conditions (52). We only need to show that if (52) fails tohold for some $k$, and if $\bar{u}$ is a $c^{\infty}$ solution of the problem (1)-(3), then there must be infinite number of solutions of problem (1) - (3). We define

$$
J=\max \left\{k \mid \operatorname{det}\left(I-\Theta_{0} \sigma_{0}^{k}\right)=0\right\} \text {, }
$$

where the maximum must arrives at a finite integer because the property (10) ensure that when $k$ is large enough the matrix $\theta_{0} \sigma_{0}^{k}$ will be small enough, and hence $\operatorname{det}\left(I-\Theta_{0} \sigma_{0}^{k}\right)$ is not zero.

Now we consider the $K-$ th augmented problem $\left(A_{K}\right)$ where $K$ is large enough such that $\left\|_{0} \sigma_{0}^{K}\right\|_{\infty}<1$. According to the properties about the minimal characterizing number [2], [3], for any given $u^{k}(0,0)(k=0,1, \ldots, k-1)$ satisfyir the compatibility conditions (38), the minimal characterizing number of problem $\left(A_{K}\right)$ is less than one [1], thus $\left(A_{K}\right)$ possesses a unique $c^{\infty}$ solution $\left(\mathrm{u}^{0}, u^{1}, \ldots, u^{K}\right)$, and by Lemma 3, we obtain a $c^{\infty}$ solution $u=u^{\circ}$ for the original problem (1) (3).

Because $\bar{u}$ is a $c^{\infty}$ solution of problem (1)-(3), certainly $\vec{u}(0,0), D \bar{u}(0,0), \ldots, D^{K-1} \vec{u}(0,0)$ satisfy all the compatibility conditions (38). Now, we take

$$
\mathrm{u}^{\mathrm{k}}(0,0)=\bar{u}^{\mathrm{k}}(0,0) \quad(\mathrm{k}<\mathrm{J})
$$

From (38),(34),(35),(54) and Lemma 2, the compatibility condition concerning $u^{J}(0,0)$ is as follows

$$
u^{\mathrm{J}}(0,0)=\Theta_{0} \sigma_{0}^{\mathrm{J}} u^{\mathrm{J}}(0,0)+\beta^{\mathrm{J}}\left(0, \bar{u}(0,0), D \bar{u}(0,0), \ldots, D^{\mathrm{J}-1} \bar{u}(0,0)\right) \text {. }
$$

Since $\operatorname{det}\left(I-\Theta_{0} \sigma_{0}^{J}\right)=0$, and $\bar{u}(0,0)$ has already been a solution of system (55) of linear equations, there is a solution subspace of (55) in 
$R^{n}$. Take $u^{J}(0,0)$ to be any element of this subspace. As for

$\mathrm{u}^{\mathrm{k}}(0,0)(\mathrm{J}<\mathrm{k} \leqq \mathrm{K}-1)$, noticing that $\operatorname{det}\left(I-\Theta_{0} \sigma_{0}^{\mathrm{k}}\right) \neq 0(\mathrm{k}>\mathrm{J})$, they can be determined uniquely by the compatibility conditions in succession. Therefore, by the above analysis, any element of the solution subspace of (55) would correspond to a $\mathrm{C}^{\infty}$ solution of the original problem (1) - (3). Besides, the correspondence is in an one-to-one way, because the particular derivative $D^{\mathrm{J}} u(0,0)$ of every solution is different to each other. Hence the original problem (1) - (3) possesses infinite solutions, and theorem 1 is proved.

From the above discussion, we also know the aystem of linear algebraic equations satisfied by $D^{k} u(0,0)$ is in the from of $(55)$, i.e.

$$
D^{k} u(0,0)=\Theta_{0} \sigma_{0}^{k} D^{k} u(0,0)+\beta^{k}\left(0, u(0,0), D u(0,0), \ldots, D^{k-1} u(0,0)\right),
$$

where $k=1,2,3, \ldots$. Therefore, all $D^{k} u(0,0)$ can be uniquely determined iff (52) is satiafied. Furthermore, by induction on $k$, we are able to show all $E D^{k-1} u, E^{2} D^{k-2} u, \ldots, E^{k-1} D u, E^{k} u$ can be expressed in terms of $D^{k} u$. Thus we may change theorem 1 into the following

Theorem 2. Under assumptions 1-5, there exists a unique local $c^{\infty}$ solution $u$ for the typical boundary value problem (1)-(3) iff all the derivatives of the unknown solution can be uniquely determined at the vertex from the equations and the boundary conditions.

Note By the analysis in the proof of theorem 1, we could imagine that the degree of freedom $d$ for the solutions of problem (1)-(3) would satisfy

$$
\mathrm{n}-\operatorname{Rank}\left(\mathrm{I}-\Theta_{0} \sigma_{0}^{\mathrm{J}}\right) \leqq \mathrm{d} \leq \sum_{\mathrm{k}=1}^{\infty}\left(\mathrm{n}-\operatorname{Rank}\left(\mathrm{I}-\Theta_{0} \sigma_{0}^{\mathrm{k}}\right)\right) .
$$

We quess that the right ineguality might turn to be an equality, and the rightest side of (57) should be less than or equal to $n$.

Note For the typical boundary value problems in functional form or with free boundary [3], a similar discussion could be given, but more complex notations are needed, and the details are not intended to be described here. Besides, for the importance of the discussion on the boundary value probleins, we refer to the previous work of the authors and the books [4] and [5].

At last, our thanks go to prof. Gu Chao-hao who mentioned us some 
basic ideas on how to weaken the solvable conditions during the period of his paper [6] on a related problem.

\section{References}

[1] Li Daqian and Yu Wenci, Fudan Journal (Natural Science) (in Chinese), 2 (1979), 84.Scientia Sintca 3 (1980), 211.

[2] Lee Da-Tsin ( $\mathrm{Li}$ Daqian) and Yu Wen-tzu ( Yu Wenci), Scientia Sinica, 13 (1964), 529.

[3] Lee Da-tsin ( Li Daqian) and Yu Wen-tzu (Yu Wenc1), Scientia Sinica, 13 (1964), 551.

[4] Courant R. and Friedrichs K.O., Supersonic Flow and Shock Waves, New York, 1948.

[5] Jeffrey A. Quasilinear Hyperbolic Systems and Waves, Pitman Pablishing, 1976.

[6] Gu Chaohao, Acta Math. Sinica (in Chinese), 13 (1963), 32. 
Imprimé en France

par

l'Institut National de Recherche en Informatique et en Automatique 
